\title{
A Non-Destructive Imaging Method Based on Integral Signals of Ultrasonic Pulse
}

\author{
Qiuhe Huang
}

Department of Mathematics and Physics, Liuzhou Institute of Technology, Liuzhou 545616, China

Corresponding Author Email: huanghe725@163.com

https://doi.org/10.18280/rces.080102

Received: 8 December 2020

Accepted: 12 February 2021

\section{Keywords:}

image processing, green function, edge detection, ultrasonic phased array, Laplace operator

\begin{abstract}
For ultrasonic phased array imaging, the most popular technique is the delay superposition algorithm of time domain signals with fixed weights. However, this technique and similar approaches cannot effectively suppress the non-scanning azimuth noise, which drags the imaging resolution. To overcome the problem, this paper proposes a nondestructive imaging method based on ultrasonic pulse integral signal, on the basis of ultrasonic phased array imaging. This method relies on the Green function to implement inverse Laplace transform on the ultrasonic pulse signal, obtains the analytical expression of the operator through mathematical derivations and calculations, and adopts the eigenvalues obtained by Laplacian matrix decomposition for image edge detection. The experimental results show that the operator is simple and effective, and better in imaging than other methods.
\end{abstract}

\section{INTRODUCTION}

Nondestructive testing is an effective and accurate means to evaluate the internal health of the structure in real time, without damaging the internal structure or performance of the material. There are many popular ways of nondestructive testing, including but not limited to X-ray, tomography, ultrasonic testing, thermal imaging, and penetration method.

Nondestructive imaging stands out among various nondestructive testing methods, because of its high sensitivity, strong penetration, flexible and convenient use, and harmlessness to human body. During ultrasonic imaging, body wave and guided wave are the common waveforms. Body wave is mainly responsible for detecting thick components. However, body wave detection is not efficient, because the transducer needs to be moved many times to detect the damage of various components. Guided wave is mainly responsible for detecting thin structures, owing to its long propagation distance and high sensitivity.

The ultrasonic wave propagating in thin plate structure is called Lamb wave. Currently, Lamb wave is mostly detected with an ultrasonic transducer array, composed of several exciting elements and receiving elements. The exciting element sends an excitation signal, which is received by the receiving element, producing the Lamb wave signal containing the internal damage features of the target component. By analyzing and processing this signal, it is possible to obtain the internal image of the structure, such that the internal state of the structure can be evaluated against the image.

Since imaging resolution is critical to evaluation effect, this paper decides to design a nondestructive imaging method based on ultrasonic pulse integral signal.

\section{LITERATURE REVIEW}

The imaging methods based on Lamb wave array mainly include time reversal imaging, migration imaging, and delay superposition imaging of phased array. Alleyne and Cawley [1] found that the time reversal algorithm can automatically compensate for the dispersion of the Lamb wave during damage detection. Giurgiutiu [2] applied the time reversal algorithm to composite materials, and improved the algorithm performance through wavelet transform. Wilcox et al. [3] combined synthetic aperture with time reversal method, and succeeded in damage imaging. Facing different isotropic media, the signals from different paths can be dealt with to compensate for the dispersion effect. During the damage imaging phase, however, the time-domain signals must be reversed before reconstruction, which adds to the computing time of the algorithm, making it less efficient in damage detection.

Migration imaging is widely adopted for damage imaging based on ultrasonic Lamb wave. Sicard et al. [4] were the first to combine migration imaging with the damage detection of plate and shell structure. Zhao et al. [5] developed a post-stack reverse time migration imaging method for damage imaging. Wright et al. [6] applied pre-stack migration imaging to detect the structural health of concrete, and successfully captured different types of damages in concrete members.

The working of phased array is realized by arranging a regular transducer array and controlling the sending and receiving of signals by specific elements. In a phased array, the exciting element sends out exciting signals, while the receiving element receives the signals. The exciting and receiving signals are delayed for a specific time to adjust the focus and reorient the beam, laying the basis for ultrasonic imaging. By load difference method, $\mathrm{Yu}$ et al. [7] compared the difference of guided wave signals under different loads, without recording the lossless Lamb wave signal. Urban et al. [8] extracted the multi-path damage scattering signals from the Lamb wave signals scattered by the multiple paths in the target structure, and processed the extracted signals by the distributed acoustic sensing (DAS) algorithm, thereby realizing the imaging of damages. 
If each receiver receives time-domain signals excited by multiple excitation sources, the signals obtained by each receiver from those sources can be combined into a multistatic matrix. Anand et al. [9] proposed a total focusing algorithm based on the full matrix capture model. The principle of the algorithm is as follows: The signals are excited separately by multiple sources, and each receiver in the array receives the excitation signals from each source; then, the multiple groups of received time-domain signals are processed by delay superposition to obtain the damage image. Hence, their algorithm can be understood as the superposition of multiple delay algorithms.

\section{LAPLACE TRANSFORM OF ULTRASONIC PULSE SIGNAL BASED ON GREEN FUNCTION}

\subsection{Laplacian graph}

Suppose there is a weighted graph $G=(V, E, W)$, with $V$ being the set of vertices, $\mathrm{E} \subseteq \mathrm{V} \times \mathrm{V}$ being the set of edges, and $W$ being the similarity matrix. Then, the weight of edge $(i, j)$ can be represented by:

$$
W(\mathrm{i}, \mathrm{j})=\left\{\begin{array}{lr}
w(i, j), & (i, j) \in E \\
0, & \text { otherwise }
\end{array}\right.
$$

Let $\mathrm{D}=\operatorname{diag}\left(d_{i} ; i \in \mathrm{V}\right)$ be a diagonal matrix, with $d_{i}=$ $\sum_{j=1}^{|V|} w(i, j)$ being the elements on the diagonal; $L=D-W$ be the non-normalized Laplacian matrix of graph. Then, the normalized Laplacian matrix $L_{n}=D^{-1 / 2} L D^{-1 / 2}$ can be defined as:

$$
L_{n}(i, j)=\left\{\begin{array}{l}
1, i=j \\
-w(i, j) / \sqrt{d_{i} d_{j}}, \quad i \neq j \text { and }(i, j) \in E \\
0, \quad \text { otherwise }
\end{array}\right.
$$

The Green function is the left inverse of the Laplace operator:

$$
G \Delta(i, j)=I(i, j)-d_{j} / \text { Vol }
$$

where, $\mathrm{Vol}=\sum_{i \in V} d_{i} ; \mathrm{I}$ is a $|V| \times|V|$ identity matrix.

The Green function can be calculated by:

$$
\mathrm{G}(i, j)=\int_{0}^{\infty} d_{i}^{1 / 2}\left(H_{t}(i, j)-u_{l}(i) u_{l}(j)\right) d_{j}^{1 / 2} d t
$$

where, $u_{l}$ is the eigenvector corresponding to the eigenvalue of zero.

The Green function can be normalized as $G_{n}=$ $D^{1 / 2} G D^{-1 / 2}$. Then, the relationship between the normalized Green function and Laplacian spectrum can be described by:

$$
G_{n}(i, j)=\sum_{k=2}^{|V|} \frac{1}{\rho_{k}} u_{k}(i) u_{k}(j)
$$

where, $\rho_{k}$ and $u_{k}$ are the eigenvalue and eigenvector of the normalized Laplacian matrix $L_{n}$.

Accordingly, the non-normalized Green function $\bar{G}$ can be obtained by:

$$
\bar{G}(i, j)=\sum_{k=2}^{|V|} \frac{1}{\sigma_{k}} v_{k}(i) v_{k}(j)
$$

where, $\sigma_{k}$ and $v_{k}$ are the eigenvalue and eigenvector of nonnormalized Laplacian matrix $L$, respectively. Obviously, the normalized Green function is the pseudo inverse of the normalized Laplacian matrix $L_{n}$ :

$$
G_{n} L_{n}=L_{n} G_{n}=I-u_{1} u_{1}^{T}
$$

The relationship among the Green function G, nonnormalized Green function $\bar{G}$, and normalized Green function $G_{n}$ can be calculated by:

$$
G \Delta=\bar{G} L=G_{n} L_{n}=L_{n} G_{n}=I
$$

\subsection{Ultrasonic pulse signal transfer function}

In the classical array signal processing, the signal is assumed as a far-field signal in most cases. When Lamb wave phased array is used in imaging detection, the wave front of the signal received by the array is spherical, when the secondorder scattering wave field, which is formed by the interaction between imaging and detection waves, propagates to the receiving element array. Therefore, the received signal belongs to the near-field data. In general, far and near fields are distinguished according to Figure 1, where $L$ is array aperture, and $\lambda$ is the wavelength of the signal.

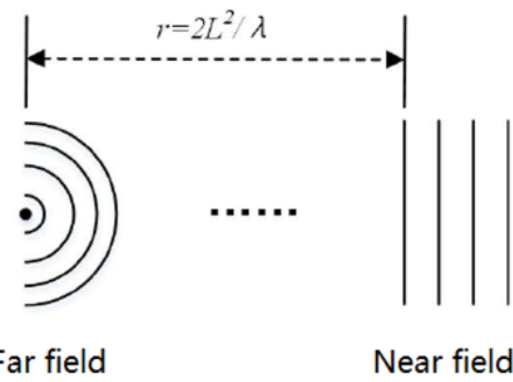

Figure 1. Distinguishing between far field and near field

In the Lamb wave near-field model, the beam pattern can be adopted to illustrate the gain of the incoming wave in different directions, under the effect of the array weight. The gain is related to the spatial coordinates $(\mathrm{x}, \mathrm{y})$ of the incoming wave. The beam pattern is shown as Figure 2 .

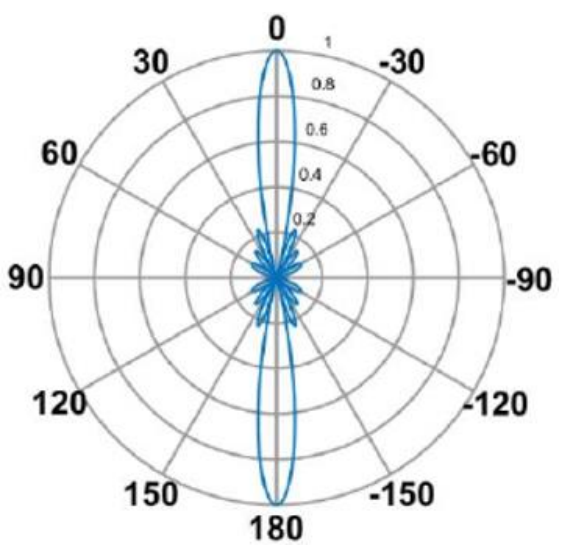

Figure 2. Beam pattern 
The ultrasonic pulse signal transfer function can be defined as:

$$
G_{u}(x)= \begin{cases}0 & x<0 \\ \frac{2}{u} \exp \left(-\frac{x}{u}\right) \sin \left(\frac{x}{u}\right) & x>0\end{cases}
$$

$G_{u}(x)$ needs to satisfy the differential equation:

$$
\frac{u^{3}}{6} G_{u}^{\prime \prime \prime}+{\frac{u^{2}}{2}}^{\prime \prime}+u^{\prime}+G_{u}=\vartheta(x)
$$

where, $\vartheta(x)$ is the Dirac shock function.

Thus, the Green function can be applied to signal differentiation. The derivative of signal $I(x)$ can be defined as:

$$
I^{\prime}(x)=\lim _{u \rightarrow 0} \frac{I(x+u)-I(x-u)}{2 u}
$$

If $I(x)$ is a boundary signal, there exist a boundary condition $\lim _{x \rightarrow \pm \infty} I(x)=0$. Then, the solution of the equation can be expressed by Green function $G_{u}(x)$ :

$$
I(x) \equiv I(x-u)=\int_{-\infty}^{+\infty} G_{u}(\gamma) I(x-\gamma) d \gamma
$$

\section{IMAGE EDGE DETECTION BASED ON EDGE EXTRACTION OPERATOR}

In the background medium, the Green function can be regarded as the time-dependent scattering wave propagating from the exciting and receiving elements to any point $\mathrm{x}$ in the whole plate space. The Green function starting from the exciting and receiving elements can be expressed as:

$$
\begin{aligned}
& g^{a}(x)=\left[g\left(x, x_{1}^{a}\right), \ldots, g\left(x, x_{N}^{a}\right)\right]^{T} \\
& g^{s}(x)=\left[g\left(x, x_{1}^{s}\right), \ldots, g\left(x, x_{M}^{s}\right)\right]^{T}
\end{aligned}
$$

According to the orthogonality of eigenvectors, the propagation conditions of the wave can be obtained as:

$$
\begin{aligned}
& g_{k}^{a} \cdot g^{a}(x)= \begin{cases}\left\|g_{k}^{a}\right\| & x=x_{k} \\
0 & x \neq x_{k}\end{cases} \\
& g_{k}^{s} \cdot g^{s}(x)= \begin{cases}\left\|g_{k}^{s}\right\| & x=x_{k} \\
0 & x \neq x_{k}\end{cases}
\end{aligned}
$$

Suppose the singular vectors related to the singular values beyond the k-th value are generated by noise. The imaging conditions can be formulated from the noise signal through multiple signal classifications, in view of the stochasticity of the noise space. In this way, it is possible to image damages at superhigh resolution.

The imaging indexes of the Lamb wave propagating from the exciting element to the damage and that propagating from the damage to the receiving element can be worked out respectively:

$$
\begin{aligned}
I^{a}(x) & =\frac{1}{\sum_{k=k+1}^{\min \{M, N\}} g_{k}^{a} \cdot g^{a}(x)} \\
I^{S}(x) & =\frac{1}{\sum_{k=k+1}^{\min \{M, N\}} g_{k}^{s} \cdot g^{s}(x)}
\end{aligned}
$$

The final super-resolution imaging indexes can be synthetized from the two sets of imaging indexes obtained by the exciting and receiving elements, respectively:

$$
I(x)=\frac{1}{\sum_{k=k+1}^{\min \{M, N\}} g_{k}^{a} \cdot g^{a}(x) \cdot \sum_{k=k+1}^{\min \{M, N\}} g_{k}^{s} \cdot g^{s}(x)}
$$

In the limit $u \rightarrow 0, D_{u}(x)$ is the impulse response of a differential operator. To improve the extraction of edges, $I(x+u)$ can be transformed into:

$$
I(x)=I\left(\frac{x}{\mu}+u\right)
$$

where, $\mu$ is a scale factor.

According to the Green functions $G_{u}(x)$ and $G_{\mu u}(\omega)$, the differential I can be estimated, and the operator can be obtained by:

$$
\begin{aligned}
D(\mathrm{x}) & =-\frac{A}{2 u}\left\{\left[\exp \left(-\frac{x}{\mu u}\right)+\exp \left(\frac{x}{\mu u}\right)\right] \sin \left(\frac{x}{\mu u}\right)\right. \\
& \left.+K\left[\exp \left(-\frac{x}{\mu u}\right)+\exp \left(\frac{x}{\mu u}\right)\right] \sin \left(\frac{x}{\mu u}\right)\right\}
\end{aligned}
$$

where, $A=a /(1+a)(\mu u)^{2} ; K=\mu^{2} / a$.

The above formula is the exact expression of the edge detection operator by the Green function. Because the digital image is processed, the differential operator must be discretized firstly, and transformed into a digital image. The algorithm can be implemented in the following steps:

Step 1. Read the grayscale image.

Step 2. Convolute each row, and calculate the operator $D(n)$ of the image. Then, convolute the integral form of $D(n)$ with the column of the image to obtain the gray gradient $g(x, y)$ and the gradient direction $\alpha /$

Step 3. Implement non-maximum suppression, that is, ensure that the boundary strength of each edge point is greater than that of the two adjacent pixels (positive and negative) along the gradient direction of that point, so as to refine the edge.

Step 4. Implement lag threshold processing and edge connection, that is, set double thresholds $\mathrm{T} 1$ and $\mathrm{T} 2$, and judge each point by the following criteria: the point with gradient value greater than $\mathrm{T} 2$ must be a boundary point, the point with gradient value smaller than $\mathrm{T} 1$ cannot be a boundary point, and whether the point between $\mathrm{T} 1$ and $\mathrm{T} 2$ is a boundary point needs to be judged according to the edge information of adjacent points.

\section{EXPERIMENT AND RESULTS ANALYSIS}

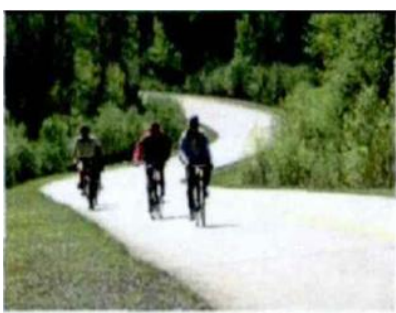

(1) Original image

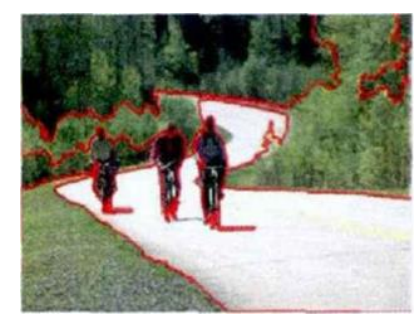

(2) Image edge detection
Figure 3. Image edge detection by our algorithm 
Table 1. Comparison of fusion performance of the three algorithms in decomposing four layers

\begin{tabular}{cccccc}
\hline Algorithm & Entropy & Cross entropy & Mutual information & RMSE & PSNR/dB \\
\hline FCM & 3.873 & 0.0377 & 4.0286 & 2.0873 & 39.485 \\
Nystrom & 3.982 & 0.0208 & 4.0372 & 1.8735 & 37.398 \\
MSNcut & 4.297 & 0.0119 & 4.1823 & 1.6209 & 41.298 \\
Our algorithm & 4.482 & 0.0047 & 4.4982 & 1.2873 & 40.872 \\
\hline Note: RMSE and PSNR are short for root mean square error and peak signal-to-noise ratio, respectively.
\end{tabular}

Table 2. Influence of fusion layers on edge detection performance

\begin{tabular}{cccccc}
\hline Number of layers & Entropy & Cross entropy & Mutual information & RMSE & PSNR/dB \\
\hline 2 & 4.387 & 0.0071 & 4.3384 & 3.1723 & 33.498 \\
3 & 4.429 & 0.0062 & 4.4109 & 2.8734 & 36.325 \\
4 & 4.482 & 0.0047 & 4.4982 & 1.2873 & 40.872 \\
5 & 4.496 & 0.0039 & 4.5287 & 1.0934 & 41.342 \\
6 & 4.592 & 0.0035 & 4.5983 & 0.8893 & 43.987 \\
7 & 4.609 & 0.0034 & 4.6002 & 0.7923 & 44.712 \\
8 & 4.617 & 0.0034 & 4.6007 & 0.5322 & 44.872 \\
\hline
\end{tabular}

To evaluate its performance, the proposed algorithm is compared with fuzzy c-means clustering (FCM) algorithm [10], Nystrom algorithm [11] and MSNcut algorithm [12]. The experimental images are all $381 \times 431$ pixels in size. Figure 3 illustrates the imaging effect of our algorithm.

Table 1 compares the fusion performance of the three algorithms in decomposing four layers. It can be found that our algorithm achieved good effect of quantitative analysis, and outshined the other methods in edge detection.

Table 2 presents the influence of fusion layers on edge detection performance. As the number of fusion layers increased, the entropy and mutual information of the fused image grew, a sign of the growth of the amount of information extracted from the fused image. As the number of fusion layers increased, the cross entropy of the fused image decreased, the RMSE of the fused image declined, and the PSNR rose. The more layers of image decomposition and fusion, the richer the details and the higher the quality of edge detection. However, more layers complicated the operation. Thus, the number of fusion layers should not be too large. When there were seven layers, the number of layers had a negligible contribution to the fusion performance, but the computing cost was very high.

The experimental results show that our method can achieve fast and accurate fusion from coarse to fine, and detect image edges accurately and efficiently.

\section{CONCLUSIONS}

Green function is a unique way to transform the ultrasonic pulse integral signal, making image edge detection more accurate. Based on ultrasonic pulse integral signal, this paper takes advantage of the spatial frequency locality, directionality, and multi-resolution of the Green function transform, adopts different fusion rules for different frequency characteristic regions of different decomposition layers to realize image edge detection. Through objective evaluation and visual perception, it is proved that the proposed method can achieve fast and accurate fusion from coarse to fine, and detect image edges accurately and efficiently.

\section{ACKNOWLEDGMENTS}

This work is supported by 2017 Basic Ability Improvement of Young and Middle-aged Teachers in Guangxi Universities
(Grant No.: 2017KY1383).

\section{REFERENCES}

[1] Alleyne, D.N., Cawley, P. (1992). Optimization of Lamb wave inspection techniques. Ndt \& E International, 25(1): 11-22. https://doi.org/10.1016/0963-8695(92)90003-Y

[2] Giurgiutiu, V. (2005). Tuned Lamb wave excitation and detection with piezoelectric wafer active sensors for structural health monitoring. Journal of Intelligent Material Systems and Structures, 16(4): 291-305. https://doi.org/10.1177/1045389X05050106

[3] Wilcox, P.D., Dalton, R.P., Lowe, M.J.S., Cawley, P. (1999). Mode and transducer selection for long range Lamb wave inspection. In Key Engineering Materials, 167: 152-161. https://doi.org/10.4028/www.scientific.net/KEM.167168.152

[4] Sicard, R., Goyette, J., Zellouf, D. (2002). A SAFT algorithm for lamb wave imaging of isotropic plate-like structures. Ultrasonics, 39(7): 487-494. https://doi.org/10.1016/S0041-624X(01)00087-7

[5] Zhao, X., Royer, R.L., Owens, S.E., Rose, J.L. (2011). Ultrasonic Lamb wave tomography in structural health monitoring. Smart Materials and Structures, 20(10): 105002 . $1726 / 20 / 10 / 105002$ http://dx.doi.org/10.1088/0964-

[6] Wright, W., Hutchins, D., Jansen, D., Schindel, D. (1997). Air-coupled Lamb wave tomography. IEEE Transactions on Ultrasonics, Ferroelectrics, and Frequency Control, $\quad 44(1)$ : $\quad$ 53-59. https://doi.org/10.1109/58.585190

[7] Yu, L., Bottai-Santoni, G., Giurgiutiu, V. (2010). Shear lag solution for tuning ultrasonic piezoelectric wafer active sensors with applications to Lamb wave array imaging. International Journal of Engineering Science, 48(10):

848-861. https://doi.org/10.1016/j.ijengsci.2010.05.007

[8] Urban, M.W., Pislaru, C., Nenadic, I.Z., Kinnick, R.R., Greenleaf, J.F. (2012). Measurement of viscoelastic properties of in vivo swine myocardium using lamb wave dispersion ultrasound vibrometry (LDUV). IEEE Transactions on Medical Imaging, 32(2): 247-261. https://doi.org/10.1109/TMI.2012.2222656

[9] Anand, C., Groves, R., Benedictus, R. (2020). A gaussian 
beam based recursive stiffness matrix model to simulate ultrasonic array signals from multi-layered media. Sensors, 20(16): https://doi.org/10.3390/s20164371

[10] Pan, R., Gao, W., Liu, J., Wang, H. (2010). Automatic detection of the layout of color yarns for yarn-dyed fabric via a FCM algorithm. Textile Research Journal, 80(12): 1222-1231. https://doi.org/10.1177/0040517509355349
[11] Liu, X., Chen, J., Xu, L. (2018). Fast Root-MUSIC Algorithm Based on Nystrom Method and Spectral Factorization. Progress in Electromagnetics Research, 78: 81-88. https://doi.org/10.2528/PIERL18060301

[12] Basavaprasad, B. (2017). Image segmentation using MSNCut algorithm. International Journal of Computer Applications, 162(2): 27-30. 